\title{
Adaptación cultural del cuestionario LiNQ (Lung Information Needs Questionnaire) en pacientes con enfermedad respiratoria crónica en un programa de rehabilitación pulmonar en Cali, Colombia
}

Cultural Adaptation of the LINQ (Lung Information Needs Questionnaire) Questionnaire in Patients with Chronic Respiratory Disease in a Pulmonary Rehabilitation Program in Cali, Colombia

Adaptação cultural do questionário uINQ (Lung Information Needs Questionnaire) em pacientes com doença respiratória crônica em um programa de reabilitação pulmonar em Cali, Colômbia

Esther Cecilia Wilches Luna, $\mathrm{F}^{\top}$, Lorena Obregón A., $\mathrm{F}^{2}$, Maria Isabel Delgado H., $\mathrm{FT}^{3}$, Diana Fernanda Rebolledo A., $\mathrm{F}^{4}$, Adiela Terreros A., $\mathrm{F}^{5}$

Recibido: 1 de marzo de $2013 \bullet$ Aprobado: 26 de noviembre de 2013

Doi: dx.doi.org/10.12804/revsalud12.1.2014.02

Para citar este artículo: Wilches EC, Obregón L, Delgado MI, Rebolledo DF, Terreros A. Adaptación cultural del cuestionario Lina (Lung Information Needs Questionnaire) en pacientes con enfermedad respiratoria crónica en un programa de rehabilitación pulmonar en Cali, Colombia. Rev Cienc Salud. 2014; 12 (1): 23-34. doi: dx.doi.org/10.12804/revsalud12.1.2014.02

Resumen

Objetivo: Adaptar culturalmente el cuestionario LINQ (Lung Information Needs Questionnaire) en pacientes con enfermedad pulmonar crónica. Métodos: Estudio descriptivo, después de la traducción del cuestionario por traductor certificado y de la adaptación cultural, se aplicó en ocho pacientes para identificar las dificultades y dudas relacionadas con el entendimiento de las preguntas, la terminología y para evaluar no solo la calidad de la traducción, sino también para verificar aspectos prácticos de su aplicación. Posteriormente, se realizó una segunda traducción al idioma inglés la cual fue enviada a los autores para asegurar el contenido original del cuestionario y conseguir el aval para su uso. Resultados: La muestra en la fase de adaptación cultural fue

\footnotetext{
Facultad de Salud. Universidad del Valle. Escuela Rehabilitación Humana, Grupo de Investigación Ejercicio y Salud Cardiopulmonar de la Universidad del Valle. Correspondencia: esther.wilches@correounivalle.edu.co

2 Facultad de Salud. Universidad del Valle.

SAF. Cali Colombia. Correspondencia: isabeladelgad33@hotmail.com

4 Fundación Joseph Pilates. Pasto, Colombia.

5 Centro de Salud Familiar (Cesfam). Lo Barnechea, Santiago de Chile.
} 
constituida por 6 hombres y 2 mujeres con una edad promedio de 61 años. La versión en español del LINQ y la adaptación cultural fue comprensible y fácil de usar y no presentó discrepancias con la versión original durante el proceso de retratraducción. Conclusiones: La traducción al español y la adaptacion del LINQ fue adecuada pues los pacientes no manifestaron dificultades para entender y responder las preguntas. Esto podrá facilitar la realización de futuros estudios que evalúen el componente educativo en los programas de rehabiltación pulmonar.

Palabras claves: rehabilitación, educación, traducción, enfermedad pulmonar obstructiva crónica.

\section{Abstract}

Objective: To perform a cultural adaptation of the LINQ (Lung Information Needs Questionnaire) for patients with chronic pulmonary disease. Method: Descriptive study. After the translation of the questionnaire by a certified translator, and after its cultural adaptation, it was applied in eight patients in order to identify the difficulties and doubts related to an understanding of the questions and of its terminology, not only to evaluate the quality of the translation, but to verify practical aspects of its application, as well. Afterward, a second translation was done from Spanish to English, and sent back to the authors to ensure the original content of the questionnaire was maintained, and to obtain endorsement for its use. Results: During the cultural adaptation phase, the sample was constituted by 6 men and 2 women, with an average age of 61 years. The Spanish version of the LINQ, as well as the cultural adaptation, was easy to use and did not show any discrepancies with the original version during the re-translation process. Conclusions: The LINQ's translation to Spanish and the cultural adaptation proved to be adequate, because the patients did not express any difficulties in understanding and answering the questions. This will facilitate future studies that evaluate the educational component in the pulmonary rehabilitation program.

Key Words: Rehabilitation, education, translating, chronic obstructive pulmonary disease.

\section{Resumo}

Objetivo: adaptar culturalmente o questionário LINQ (Lung Information Needs Questionnaire) em pacientes com doença pulmonar crônica. Métodos: Estudo descritivo, depois da tradução do questionário por tradutor certificado e da adaptação cultural, aplicou-se a oito pacientes para identificar as dificuldades e dúvidas relacionadas com o entendimento das perguntas, a terminologia e para avaliar não só a qualidade da tradução, más também para verificar aspectos práticos de sua aplicação. Posteriormente, realizou-se uma segunda tradução ao idioma inglês a qual foi enviada aos autores para assegurar o conteúdo original do questionário e conseguir o aval para seu uso. Resultados: a amostra na fase de adaptação cultural foi constituída por 6 homens e 2 mulheres com uma idade média de 61 anos. A versão em espanhol de LINQ e a adaptação cultural foram compreensíveis e fáceis de usar e não apresentaram discrepâncias com a versão original durante o processo de retradução. Conclusões: a tradução ao espanhol e a adaptação do LINQ foi adequada pois os pacientes não manifestaram dificuldades para entender e responder as perguntas. Isto poderá facilitar a realização de futuros estudos que avaliem o componente educativo nos programas de reabilitação pulmonar.

Palavras-chave: reabilitação, educação, tradução, doença pulmonar obstrutiva crônica. 


\section{Introducción}

La rehabilitación pulmonar (RP) es un recurso terapéutico no farmacológico, dirigido a pacientes con enfermedad pulmonar crónica. Sin embargo, a pesar de los avances en el conocimiento y en la práctica de la rehabilitación pulmonar, los estudios que evalúen el conocimiento que los pacientes tienen acerca de su enfermedad son escasos en nuestro medio.

Basados en una creciente evidencia científica, los principales objetivos de la RP son (1): reducir los síntomas, mejorar la calidad de vida y aumentar la participación física y emocional de los pacientes en las actividades cotidianas. Los componentes de un programa de RP incluyen el manejo médico, la educación, el apoyo emocional, el condicionamiento físico, el entrenamiento respiratorio y el apoyo nutricional (2).

El componente educativo se incluye con el objetivo de optimizar las habilidades de los pacientes, la capacidad de sobrellevar la enfermedad y mejorar el estado de salud, además de la cesación del hábito tabáquico. La educación se extiende a todos los componentes de la RP y es una responsabilidad compartida entre pacientes, familiares y profesionales de la salud. La evaluación del valor de la educación puede ser difícil debido al tiempo requerido y a las dificultades para medir la contribución directa en los resultados; esto conlleva a que el rol de la educación en las enfermedades pulmonares crónicas no haya sido tradicionalmente estudiado y medido en investigaciones clínicas (3). Con el fin de optimizar el papel del componente educativo en los programas de RP y la adherencia en el autocuidado de la enfermedad, el personal de salud debe desarrollar diferentes estrategias y competencias que le faciliten identificar las necesidades educativas de los pacientes que padecen enfermedades pulmonares crónicas. En este sentido, el cuestionario LINQ (Lung Information Needs Questionnaire) aparece como una herramienta práctica para detectar áreas en las cuales el paciente requiere educación y es sensible a los cambios que se puedan presentar después de la RP. Esto permite identificar los temas realmente necesarios para la educación del paciente y diseñar programas personalizados $(4,5)$.

El LINQ se desarrolló utilizando un procedimiento interactivo con 10 grupos focales conformados por 59 pacientes, los cuales participaron en la construcción de los ítems del cuestionario. Mide la perspectiva del paciente, la necesidad individual de educación y el impacto de la iniciativa de capacitación de los programas de RP; es decir, la calidad del componente de formación, y puede ser considerado una medida rutinaria de los resultados de la RP (6).

El LINQ ha sido traducido del inglés a los idiomas de países que se han unido a la iniciativa de poner en práctica el cuestionario. Sin embargo, para su utilización en Colombia es necesario la traducción y adaptación cultural, para que sea útil y se pueda convertir en una herramienta para complementar la evaluación e intervención de los pacientes con enfermedad respiratoria crónica en los programas de RP. El objetivo de este estudio, fue realizar la traducción del LINQ al español y su adaptación cultural.

\section{Materiales y métodos}

Estudio descriptivo realizado en ocho pacientes con EPOC; se consideró una investigación sin riesgo (Resolución 8430 de 1993 del Ministerio de Salud). El proyecto fue aprobado, por el Comité de Investigación en la institución donde se llevó a cabo la recolección de la información y por el Comité Técnico del Servicio de RP. Se incluyeron los pacientes que cumplieron con los criterios de ingreso establecidos por el programa de RP de la institución y quienes estuvieron de acuerdo con participar y firmaron el consentimiento informado y al mismo tiempo tuvieron capacidad para responder al cuestionario LINQ. El trabajo se realizó en el Hospital Universitario Evaristo García, de nivel III de complejidad, en la ciudad de Cali. 
El LINQ consta de 16 preguntas cerradas con múltiple respuesta, divididas en seis dominios (tabla 1); solamente tiene una pregunta abierta, sin puntuación, con el objetivo de aportar información sobre inquietudes o comentarios acerca de la enfermedad pulmonar de cada paciente; $y$ dos preguntas para codificarse con propósitos demográficos pero no como parte del cuestionario LINQ; lo que da un total de 19 preguntas. Para calcular el puntaje por dominios, para todos, excepto para 'Fumador' (preguntas 10, 11 y 12), se sumarán los puntajes en relación con las opciones marcadas correspondientes a las preguntas. Para el dominio 'Fumador', si el paciente obtuvo 0 para la pregunta 10 (p. ej. ya no es fumador crónico) entonces el puntaje para el área será 0 independientemente de las respuestas dadas a las preguntas 11 y 12 . Si el paciente obtuvo 1 a la pregunta 10, entonces se sumarán los puntajes de las preguntas 10 a 12. El puntaje mínimo (necesidad de información baja) es 0 y el puntaje máximo (necesidad de información alta) es 25 . El tiempo para realizar la prueba fue de 6 minutos aproximadamente.

Tabla 1. Instrucciones de Puntuación por cada dominio del cuestionario LINQ

\begin{tabular}{clc}
\hline Preguntas & \multicolumn{1}{c}{ Dominio } & $\begin{array}{c}\text { Rango de } \\
\text { puntuación }\end{array}$ \\
\hline $1-4$ & $\begin{array}{l}\text { Conocimiento de } \\
\text { la enfermedad }\end{array}$ & $0-4$ \\
$5-7$ & Medicamentos & $0-5$ \\
$8-9$ & Auto cuidado & $0-6$ \\
$13-15$ & Ejercicio & $0-5$ \\
16 & Dieta & $0-2$ \\
\hline
\end{tabular}

Para la realización de la fase de adaptación cultural, se realizó una muestra de ocho pacientes, siguiendo las orientaciones y las directrices de otros estudios que han realizado procesos parecidos $(7,8)$. Inicialmente, el investigador principal del estudio, de manera escrita solici- tó el aval a los autores del cuestionario para el uso, traducción y adaptación cultural del instrumento. Una vez obtenido, el cuestionario fue traducido por un profesional certificado. La primera versión en español fue sometida a un proceso de adaptación cultural por un comité de expertos (dos fisioterapeutas con especialización en RP y más de cinco años de experiencia en el área, un neumólogo y los autores del cuestionario original). Esta versión fue aplicada y discutida con ocho pacientes con Enfermedad Pulmonar Obstructiva Crónica (EPOC) para evaluar no solo la calidad de la traducción, sino también para verificar aspectos prácticos de su aplicación. Uno de los investigadores del estudio realizó las preguntas y anotaba las respuestas dadas por cada uno de los pacientes.

Las dudas generadas por los pacientes fueron analizadas con el grupo investigador y dos especialistas en el área, y de esta manera se generó una nueva versión del cuestionario LINQ. Después de este proceso, con las adaptaciones realizadas, la versión en español fue traducida al inglés por un segundo traductor certificado en lengua inglesa que no tenía conocimiento del cuestionario. La versión final en inglés fue comparada con la versión original para asegurar que conservara el contenido original. Esta se envió a los autores del cuestionario quienes aprobaron los cambios, el proceso realizado y enviaron el aval para su utilización. La versión final en español (anexo1) está disponible en: http://www.linq.org.uk/PDFquestionnaires/LINQspanish.pdf.

\section{Resultados}

La muestra en la fase de adaptación cultural fue constituida por 6 hombres $(75 \%)$ y 2 mujeres $(25 \%)$, con media de edad de 61 años, con diagnóstico de EPOC entre moderado y severo.

Durante la fase de adaptación cultural los ocho pacientes refirieron que entendían las preguntas $2,3,5,7,11,12,13$, 14; pero no encon- 
traron una opción de respuesta. Las opciones en el cuestionario original hacen referencia a 'doctor' o 'nurse'; en todas las preguntas donde originalmente la opción de respuesta era 'nurse' se sugirió la opción de 'fisioterapeuta'.

En la versión original del LINQ, la pregunta 9: ¿Le han dicho cuando debería llamar una ambulancia si su respiración empeora? (Have you been told when you should call an ambulance if your breathing worsens?). A esta frase se le agregaron las palabras ir a urgencias, quedando la pregunta $¿$ Le han dicho cuando debería llamar una ambulancia o ir a urgencias si su respiración empeora?

La pregunta 17: ¿ Tiene preguntas o comentarios sobre su enfermedad pulmonar? 'Si es así, escríbalas en el espacio a continuación', no fue respondida por ninguno de los pacientes durante la fase de adaptación cultural.

Los autores del LINQ aprobaron todos los cambios y la nueva traducción, sin manifestar objeciones y correcciones. La versión final incorporó todos los cambios descritos anteriormente.

\section{Discusión}

Este estudio mostró que la traducción para el idioma español y la adaptación cultural del LINQ fue adecuada pues los pacientes comprendieron bien las preguntas, como también que el instrumento es de fácil y rápida aplicación en pacientes con EPOC.

El LINQ ha sido traducido, adaptado culturalmente y utilizado en estudios en varios países lo que facilita la comparación entre los estudios realizados (5).

La versión en español del LINQ fue adaptada culturalmente, para su uso en nuestro medio conforme a las directrices actuales y teniendo en cuenta la metodología publicada de algunos autores $(9,10)$.

Este proceso es un procedimiento riguroso que implica más que una simple traducción, una vez que cada ítem debe ser adaptado culturalmente para preservar el significado conceptual del instrumento original.

La metodología aplicada en este estudio, garantizó la equivalencia técnica y semántica entre la versión original y la versión en español, poniendo a disposición un instrumento que mide objetivamente el grado de necesidad de información educativa del paciente brindada por el profesional de salud y cómo es percibida por el paciente.

Durante la fase de adaptación, en las preguntas que hacían mención a la participación de la enfermera, se sustituyó por fisioterapeuta, porque la realidad del programa donde se aplicó el cuestionario es que estos profesionales son quienes realizan las actividades de educación en rehabilitación pulmonar.

En la pregunta 9, para la adaptación cultural, los autores originales sugirieron incluir la frase 'ir a urgencias', dado a que en nuestro medio la mayoría de los pacientes no tienen la facilidad para llamar al servicio de ambulancia.

Al final del proceso observamos que no hubo necesidad de grandes adaptaciones culturales.

La literatura revisada soporta el uso del LINQ como un instrumento que puede identificar la necesidad de educación de los pacientes con enfermedades pulmonares crónicas, su versión en español, podrá contribuir con la instauración de estrategias educativas individualizadas y grupales, enfocadas a mejorar la calidad de vida de los pacientes que asisten a un programa de rehabilitación pulmonar $(11,13)$.

Los autores de este estudio identificaron algunas limitaciones en el diligenciamiento del cuestionario, debido a que algunos pacientes no sabían leer ni escribir, por tal motivo, después de la discusión con los autores originales del cuestionario y el grupo investigador, se avaló el uso del cuestionario de manera autoadministrada. Según los autores, el LINQ puede ser 
realizado de esta manera sin perder la sensibilidad del cuestionario.

Este estudio permitió concluir que, teniendo en cuenta que la mayoría de los instrumentos han sido desarrollados y validados en la lengua inglesa, para que puedan ser usados en nuestro medio, es necesario realizar procesos rigurosos de adaptación cultural. La versión final del LINQ para el español resultó ser de fácil comprensión para los pacientes y de rápida aplicación.

Sugerimos futuros estudios que analicen la fiabilidad y validez del LINQ en pacientes con enfermedad respiratoria crónica en los programas de rehabilitación pulmonar.

\section{Agradecimientos}

Las autoras agradecen a los doctores Rupert Jones y Michael Hyland por crear una herramienta útil y permitir aplicarla en su investigación. A la Unidad de Medicina Física y Rehabilitación del Hospital Universitario del Valle y a todos los profesionales y pacientes que hacen parte del Programa de Rehabilitación Pulmonar.

\section{Fuentes de financiación}

Esta investigación fue financiada por las autoras de la misma y no hubo aportes externos de fuentes de financiación.

\section{Referencias}

1. American Thoracic Society. Dyspnea Mechanisms, Assessment, and Management: a Consensus Statement. Am J Respir Crit Care Med. 1999;159(1):321-40.

2. Ries AL, Bauldoff GS, Carlin BW, Casaburi R, Emery CF, Mahler DA et al. Pulmonary Rehabilitation: Joint ACCP/AACVPR Evidence-Based Clinical Practice Guidelines. Chest. 2007;131(5 Suppl):4S-42S.

3. Rabe KF, Hurd S, Anzueto A, Barnes P, Buist S, Calverley P et al. Global Strategy for the diagnosis, management, and prevention of COPD: GOLD executive summary. Am J Respir Crit Care Med. 2007;176(6):532-55.

4. Wakabayashi R, Motegi T, Yamada K, Ishii T, Jones RCM, Hyland ME et al. Efficient Integrated Education for Older Patients with Chronic Obstructive Pulmonary Disease Using the Lung Information Needs Questionnaire. Geriatr Gerontol Int. 2011;11:422-430.

5. Sauro A, Greco A, Lo Greco P, Scalzitti F, Sirignano AR, Sortino D, et al. The copd Italian Lung Information Needs Questionnaire (LINQ): Development, Preliminary Validation, and Findings. Eur J Gen Pract. 2008;14(2):65-7.

6. Jones RCM, Hyland ME, Hanney KE. Preliminary Data from the Lung Information Needs Questionnaire (LINQ). Prim Care Respir J. 2005;14(5):270-1.

7. Gonçalves de Souza TM, Menezes PM, Leila Marques LJ, Nazário NO, Cristiane Cinara RC, Chiaradia PM, Pizzichini E. The Asthma Control Scoring System: Translation and Cross-cultural Adaptation for Use in Brazil. J Bras Pneumol. 2010;36(6):683-92.

8. Calheiros da Silva PN, Jardim JR, Da Costa e Souza GE, Michael E Hyland ME, Nascimento OA. Cultural Adaptation and Reproducibility of the Breathing Problems Questionnaire for Use in (Lung Information Needs Questionnaire) Patients with COPD in Brazil. J Bras Pneumol. 2012;38(3):339-45.

9. Tavares LA, Barreto-Neto J, Jardim JR, Souza GMC, Hlatky MA, Nascimento AO. Cross-cultural Adaptation and Assessment of Reproducibility of the Duke Activity Status Index for COPD Patients in Brazil. J Bras Pneumol. 2012;38(6):684-91.

10. Tavares LA, Barreto-Neto J, Jardim JR, Souza GMC, Hlatky MA, Nascimento AO. Cross-cultural Adaptation and Assessment of Reproducibility of the Duke Activity Status Index for COPD Patients in Brazil. J Bras Pneumol. 2012;38(6):684-91. 
11. Wilson JS, O’Neill B, Reilly J, MacMahon J, Bradley JM. Education in Pulmonary Rehabilitation: the Patient's Perspective. Arch Phys Med Rehabil. 2007;88(12):1704-9

12. Hyland ME, Jones RCM, Hanney KE. Information needs in COPD patients - the LINQ. Airways J. 2005;3:142-4.

13. Hyland ME, Jones RCM, Hanney KE. The Lung Information Needs Questionnaire: Development, Preliminary Validation and Findings. Respir Med. 2006;100(10):1807-16.

\section{Anexo}

\section{LINQ \\ Lung Information Needs Questionnaire}

\section{Instrucciones de Puntuación para el Cuestionario de Necesidad de Información Pulmonar (LINQ)}

El cuestionario mide objetivamente el grado de necesidad de información educativa del paciente brindada por el profesional de salud y como es percibida por el paciente. Se valora para producir un puntaje total en seis dominios.

A continuación se describe el cuestionario con los puntajes que se obtienen en relación con las diferentes opciones de respuesta.

2. Para producir el puntaje por dominios, para todas ellas excepto para "Fumador", sume los puntajes en relación con las opciones marcadas correspondientes a las siguientes preguntas.

\begin{tabular}{|l|l|l|}
\hline Preguntas & Dominio & Rango de Puntaje \\
$1-4$ & Conocimiento de la enfermedad & $0-4$ \\
$5-7$ & Medicamentos & $0-5$ \\
$8-9$ & Auto-Control & $0-6$ \\
$13-15$ & Ejercicio & $0-5$ \\
16 & Dieta & $0-2$ \\
\hline
\end{tabular}

Para el dominio "Fumador", si el paciente obtiene 0 para la pregunta 10 (p. ej. ya no es fumador crónico) entonces el puntaje para el área es 0 independientemente de las respuestas a las preguntas 11 y 12 . Si el paciente obtiene 1 a la pregunta 10 , entonces sume los puntajes de las preguntas 10-12, por ejemplo,
$10-12$
Fumador

Para producir el puntaje total, sume los puntajes de las seis áreas:

El puntaje mínimo (necesidad de información baja) es 0

El puntaje máximo (necesidad de información alta) es 25

Nota: Puntajes altos indican necesidad de información alta

Nota: Tiempo para realizar la prueba: 6 minutos aproximadamente 


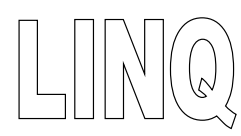

1. ¿Sabe el nombre de su enfermedad pulmonar?

Si

0

No

1

2. ¿Le ha informado su doctor o fisioterapeuta de que manera la enfermedad afecta a sus pulmones?

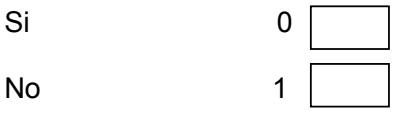

3. ¿Le ha informado su doctor o fisioterapeuta qué puede pasar en el futuro, como consecuencia de su enfermedad?

0

No

1

4. ¿Cuál de las siguientes situaciones describe lo que le ocurriría en los próximos años? MARQUE SÓLAMENTE UNA

Voy a empeorar

0

Ahora que mi enfermedad está siendo tratada, probablemente

1 seguiré igual

Ahora que mi enfermedad está siendo tratada, probablemente mejoraré.

No tengo idea

1

1 
5. ¿Le ha explicado su doctor o fisioterapeuta la razón para usar su inhalador y tomar sus medicamentos?

$\begin{array}{lll}\text { Si } & 0 & \square \\ \text { No } & 1 & \square\end{array}$

6. ¿Intenta usar su inhalador o tomar sus medicamentos exactamente como le ha recetado su médico?

$\begin{array}{ll}\text { Si } & 0 \square \\ \text { No } & 1 \square\end{array}$

7. ¿Está satisfecho con la información que sus doctores y fisioterapeutas le han dado sobre sus inhaladores y medicamentos? MARQUE SOLAMENTE UNA OPCIÓN

Entiendo todo lo que necesito saber

Entiendo todo lo que me han dicho pero me gustaría saber más

Estoy un poco confundido respecto a mis medicamentos

Estoy muy confundido respecto a mis medicamentos
0

1

2

3

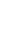

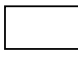

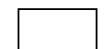

8. ¿Qué situación describe de la mejor manera lo que le han indicado hacer si su respiración empeora (ej. toma dos puff en lugar de uno)? MARQUE SOLAMENTE UNA OPCIÓN

Me han dicho que hacer y el doctor/fisioterapeuta me ha dado instrucciones escritas

0

Me han dado indicaciones pero no escritas

1

No me han dicho pero sé que hacer

No me han dicho y no sé que hacer

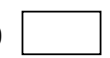

3

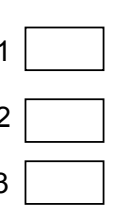


Wilches-Luna EC, Obregón-A. L, Delgado-H. MI, Rebolledo-A. DF, Terreros-A. A

LINQ

Lung Information Needs Questionnaire

9. ¿Le han dicho cuando debería llamar una ambulancia o ir a urgencias, si su respiración empeora? MARQUE SOLAMENTE UNA OPCIÓN

Me han dicho que hacer y el doctor/fisioterapeuta

me ha dado instrucciones escritas

0

Me han dicho que hacer pero no en forma escrita

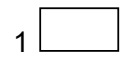

No me han dicho, pero sé que hacer

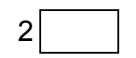

No me han dicho y no estoy seguro cuando debería llamar una ambulancia

3

10. ¿Cuál de las siguientes situaciones describe mejor su condición? MARQUE SOLAMENTE UNA OPCIÓN

Nunca he fumado (vaya a la pregunta 13)

Solía fumar pero ya no (vaya a la pregunta 13)

0

Todavía fumo (vaya a la pregunta 11)

0

11. ¿Le ha aconsejado su doctor o fisioterapeuta dejar de fumar?

$\begin{array}{ll}\text { Si } & 0 \square \\ \text { No } & 1 \square\end{array}$

12. ¿Le ha ofrecido su doctor o fisioterapeuta ayuda para dejar de fumar?

(Ej. ¿Le ha suministrado chicles con nicotina o parches, o una remisión a una clínica para dejar de fumar?)

$\begin{array}{ll}\text { Si } & 0 \square \\ \text { No } & 1 \square\end{array}$


Adaptación cultural del cuestionario LINQ (Lung Information Needs Questionnaire) en pacientes con enfermedad respiratoria crónica en un programa de rehabilitación pulmonar en Cali, Colombia

13. ¿Le ha dicho su doctor o fisioterapeuta que practique una actividad física (ej. caminar, marchar y otras formas de ejercicio?

$\begin{array}{ll}\text { Si } & 0 \square \\ \text { No } & 1 \square\end{array}$

14. ¿Le ha dicho su doctor o fisioterapeuta cuánto ejercicio físico (caminar, marchar y otras formas de ejercicio) debe hacer?
Si y sé que hacer
Si pero no estoy seguro de que hacer
Si pero no puedo hacerlo

No

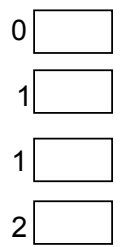

0

1

2

Hago cierto esfuerzo

La menor posible

16. ¿Que le ha dicho su doctor sobre su dieta o lo que debería comer? (Marque todas las que apliquen)

Si no le han dicho nada dé 2 puntos; si le han dicho que adelgace o gane peso dé 1 punto; si come pequeñas comidas al día, entonces el puntaje es 0 puntos independientemente de lo demás)

Tome varias comidas al día

"(Ej.: 6 comidas pequeñas al día en vez de 3 comidas grandes por día)"

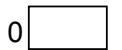


Wilches-Luna EC, Obregón-A. L, Delgado-H. MI, Rebolledo-A. DF, Terreros-A. A

LINQ

Lung Information Needs Questionnaire

Pierda o gane peso

Coma saludablemente

Nada

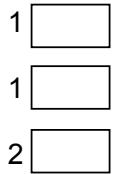

17. ¿Tiene preguntas o comentarios sobre su enfermedad pulmonar?

"Si es así, escríbalas en el espacio a continuación"

(Sin puntuación)

18. Sexo (elimine una)

Hombre $=0$

Mujer $=1$

(Para codificarse con propósitos demográficos pero no como parte de LINQ)

19. ¿En que año nació?

19

(Para codificarse con propósitos demográficos pero no como parte de LINQ

- codifique la fecha real)

34 / Rev. Cienc. Salud. 12 (1): 23-34 